Chirurg 2022 93 (Suppl 2):S142

https://doi.org/10.1007/s00104-022-01572-4

Online publiziert: 17. Januar 2022

(c) Der/die Autor(en) 2022

\section{Erratum zu: Robotische Hernienchirurgie II}

\section{Robotische primär ventrale und inzisionale Hernienversorgung (rv-TAPP und r-Rives/r-TARUP). Videobeitrag und Ergebnisse einer Kohortenstudie an 118 Patienten}

Johannes Baur ${ }^{1} \cdot$ Michaela Ramser ${ }^{1}$. Nicola Keller ${ }^{2} \cdot$ Filip Muysoms $^{3} \cdot$ Jörg Dörfer ${ }^{4}$. Armin Wiegering ${ }^{4} \cdot$ Lukas Eisner $^{1} \cdot$ Ulrich A. Dietz ${ }^{1}$

'Klinik für Viszeral-, Gefäss- und Thoraxchirurgie, Kantonsspital Olten, Olten, Schweiz

${ }^{2}$ Klinik für Allgemein-, Viszeral- und Gefässchirurgie, Kantonsspital Baden, Baden, Schweiz

${ }^{3}$ Department of Surgery, AZ Maria Middelares, Gent, Belgien

${ }^{4}$ Klinik und Poliklinik für Allgemein-, Viszeral-, Transplantations-, Gefäß- und Kinderchirurgie, Universitätsklinikum Würzburg, Würzburg, Deutschland

\section{Erratum zu:}

Chirurg 2021

https://doi.org/10.1007/s00104-02101450-5

Dieser Beitrag zum Thema „Robotische Hernienchirurgie. Rv-TAPP und r-Rives/rTARUP“ ist der zweite Teil einer Reihe von mehreren Beiträgen zum Thema. Titel und Untertitel wurden angepasst. Die Originalversion dieses Beitrags wurde korrigiert.

\section{Korrespondenzadresse}

\section{Prof. Dr. med. Armin Wiegering}

Klinik und Poliklinik für Allgemein-, Viszeral-,

Transplantations-, Gefäß- und Kinderchirurgie, Universitätsklinikum Würzburg Oberdürrbacher Straße 6, 97080 Würzburg, Deutschland wiegering_a@ukw.de

\section{Prof. Dr. med. Ulrich A. Dietz}

Klinik für Viszeral-, Gefäss- und Thoraxchirurgie, Kantonsspital Olten

Baslerstrasse 150, 4600 Olten, Schweiz

ulrich.dietz@spital.so.ch

Open Access. Dieser Artikel wird unter der Creative Commons Namensnennung 4.0 International Lizenz veröffentlicht, welche die Nutzung, Vervielfältigung, Bearbeitung, Verbreitung und Wiedergabe in jeglichem Medium und Format erlaubt, sofern Sie den/die ursprünglichen Autor(en) und die Quelle ordnungsgemäß nennen, einen Link zur Creative Commons Lizenz beifügen und angeben, ob Änderungen vorgenommen wurden.

Die in diesem Artikel enthaltenen Bilder und sonstiges Drittmaterial unterliegen ebenfalls der genannten Creative Commons Lizenz, sofern sich aus der Abbildungslegende nichts anderes ergibt. Sofern das betreffende Material nicht unter der genannten Creative Commons Lizenz steht und die betreffende Handlung nicht nach gesetzlichen Vorschriften erlaubt ist, ist für die oben aufgeführten Weiterverwendungen des Materials die Einwilligung des jeweiligen Rechteinhabers einzuholen.

Weitere Details zur Lizenz entnehmen Sie bitte der Lizenzinformation auf http://creativecommons.org/ licenses/by/4.0/deed.de.
Die Online-Version des Originalartikels ist unter https://doi.org/10.1007/s00104-021-01450-5 zu finden. 